\title{
Diminutive colorectal polyp resection comparing hot and cold snare and cold biopsy forceps polypectomy. Results of a pilot randomized, single-center study (with videos) ${ }^{1}$
}

Authors

Institutions
Victoria Gómez ${ }^{1}$, Raul J. Badillo', Julia E. Crook ${ }^{2}$, Murli Krishna ${ }^{3}$, Nancy N. Diehl², Michael B. Wallace ${ }^{1}$

${ }^{1}$ Division of Gastroenterology and Hepatology, Department of Medicine, Mayo Clinic, Jacksonville, FL, USA

${ }^{2}$ Division of Biomedical Statistics and Informatics, Department of Health Sciences Research, Mayo Clinic, Jacksonville, FL, USA

${ }^{3}$ Department of Pathology, Mayo Clinic, Jacksonville, FL, USA submitted 16. July 2014 accepted after revision 26. August 2014

\section{Bibliography}

Dol http://dx.doi.org/

10.1055/s-0034-1390789

Published online: 19.11.2014

Endoscopy International Open 2015; 03: E76-E80

(c) Georg Thieme Verlag KG

Stuttgart · New York

E-ISSN 2196-9736

\section{Corresponding author}

Victoria Gómez. MD

Department of Medicine

Mayo Clinic

4500 San Pablo Rd. South

Jacksonville, FL 32224

USA

Fax: +1-904-953-6225

gomez.victoria@mayo.edu
Background: The optimal method of diminutive polypectomy $(<6 \mathrm{~mm})$ is unknown.

Objective: To assess the rates of incomplete resection of diminutive polyps of the colon using three standard polyp resection techniques (hot snare, cold snare, and cold biopsy forceps).

Design: Randomized, pilot study.

Settings: Single-center endoscopy center.

Patients: Patients undergoing routine outpatient colonoscopies.

Interventions: Polypectomy was performed using the method to which the patient was randomized. Following retrieval of the polyp, the polypectomy base was lifted by submucosal injection of normal saline and then excised using the cold snare device. If no tissue could be removed, then at least four cold biopsies using forceps of the remaining margin were obtained.

Main outcome measures: Adequacy of resection of diminutive polyps, which was defined as no visible adenoma or hyperplastic tissue seen in the base tissue on histology.

\section{Introduction}

$\nabla$

Colonoscopy is one of the most commonly performed endoscopic procedures, and it has played a pivotal role in the detection and prevention of colorectal cancer (CRC). Small $(6-9 \mathrm{~mm})$ and diminutive $(1-5 \mathrm{~mm})$ colorectal polyps account for

${ }^{1}$ Contributorship: conception and design, V. G., R. J. B., J. E. C., M. K., N. N. D., M. B. W.; analysis and interpretation of data, V. G., J. E. C., M. K., N. N. D., M. B. W.; drafting of the article and revising it critically for important intellectual content, V. G., R. J. B., J. E. C., M. B. W.; final approval of the version to be published, V. G., R. J. B., J. E. C., M. K., N. N. D., M. B. W.

Funding sources: funded by a grant from Boston Scientific Corporation, Natick, MA, USA. It has not been previously presented.

Manuscript was presented as a poster at Digestive Disease Week, 6 May 2014; Chicago, IL, USA.
Results: A total of 60 patients were enrolled (57\% male), the mean age was 60 (range $33-82$ ), and 62 polyps were randomized from 37 patients. The mean polyp size was $3.6 \mathrm{~mm}$ (range $2-5 \mathrm{~mm}$ ) and 37 polyps (60\%) were adenomatous. Overall incomplete polyp resection rate was $9 \%(95 \% \mathrm{CI}$ $3-19 \%), 5$ of 37 (14\%) for adenomas. By the study arm, the incomplete resection rates were 1 of 18 (6\%) for hot snare, 2 of 21 (10\%) for cold snare, and 2 of 18 (11\%) for cold biopsy forceps. The majority of polyp bases were removed with cold biopsy forceps since most of the endoscopists did not feel that the saline lift cold snare method was feasible or appropriate.

Limitations: Small sample size; endoscopic mucosal resection (EMR) of the polyp base tissue was not routinely performed.

Conclusions: Recruiting patients to a pilot study that randomized polyps to one of three common methods of polypectomy for diminutive polyps was feasible, and approximately 1 in 10 diminutive polyps found on colonoscopy were incompletely resected by standard polypectomy methods.

a large proportion of lesions found during colonoscopy. Recent evidence suggests that colonoscopy may be less effective at CRC prevention than previously thought $[1,2]$. Potential reasons for the gap in CRC prevention include failure to detect polyps, rapidly growing polyps, and incomplete polyp removal [3]. Despite their widespread use, little is known about the best methods of polypectomy in terms of optimizing completeness of removal.

Many techniques are now available for polypectomy, including hot snare, cold snare, and cold biopsy forceps (including jumbo forceps). Guidelines on the standard of care for management of diminutive polyps are lacking. Several prospective studies have evaluated the completeness of polypectomy on diminutive lesions and have compared several techniques; however, these studies 
only evaluated the complete resection of one or two methods. Furthermore, highly variable estimated rates of incomplete diminutive polyp resection were found $(10-61 \%)$ [4-6]. Thus, there is a critical need to define the best method of polyp resection.

Our long-term goal is to conduct a large randomized trial to compare the rates of incomplete resection of diminutive colon polyps using three standard polyp resection techniques (hot snare, cold snare, and standard cold biopsy forceps). At the time of this study, it was understood that it would not be feasible to make any meaningful comparisons among the incomplete resection rates of the techniques in this investigation due to the intentionally small sample sizes. The primary aim was to demonstrate the feasibility of recruiting patients into a trial where they would be randomized to receive one of three methods of diminutive polyp resection using hot snare, cold snare, and cold biopsy forceps polypectomy techniques during routine outpatient colonoscopy. The secondary aim was to obtain initial rough estimates of the rate of complete resection of diminutive polyps using the three polyp resection techniques at this institution to facilitate power and sample size calculations for a larger trial. Finally, our intent was to obtain the initial estimates of incomplete resection rates to aid in refining the design of a larger future study that will follow this small pilot trial.

\section{Materials and methods}

$\nabla$

This was a single-center pilot study approved by the institutional review board (IRB) (IRB\# 12-003418) of the Mayo Clinic. In this study, the method of polypectomy was randomized to one of three techniques of diminutive $(<6 \mathrm{~mm})$ polyp resection: hot snare, cold snare, and cold biopsy forceps polypectomy.

Patients undergoing routine outpatient screening or surveillance colonoscopies were enrolled prospectively. All patients received a bowel purge consisting of a polyethylene glycol (PEG) solution, administered in either split-dose (i.e., p.m. a.m. dosing) or nightbefore fashion. Informed consent using a study protocol consent form was reviewed with the patient, and the patient's signature was obtained before commencement of the colonoscopy. Patients were excluded if they were younger than age 18 , were on anticoagulation therapy at the time of colonoscopy, were pregnant, or if they had a history of colon cancer, familial polyposis syndrome, inflammatory bowel disease, or an incomplete colonoscopy. Sedation was provided with propofol administered by the clinical nurse anesthetist, or with a combination of midazolam, fentanyl, and meperidine given by the performing endoscopist. Olympus 180 or 190 series colonoscopes (Olympus America Inc., Center Valley, PA, USA) were used. Bowel preparation using a PEG base was administered as a split dose, with the exception of earlymorning cases where administration the evening before was an option.

Four selected endoscopists with prior successful participation in colonoscopy clinical trials were chosen for participation. A teach-

\section{Video 1}

Removal of one diminutive polyp with hot snare polypectomy technique.

online content including video sequences viewable at:

www.thieme-connect.de ing tutorial that demonstrated the proper recommended technique for each method of polypectomy was first reviewed with the participating group. Randomization was set up by the statistician with a secure web interface. When a diminutive polyp was detected on routine colonoscopy, the study coordinator obtained the randomization assignment for a given polyp. The polyp size and histology were first assessed. Traditionally, the diameter between the two jaws of standard biopsy forceps is $7 \mathrm{~mm}$. Following this, polypectomy was performed using the method to which the patient was randomized:

1. Hot snare: A snare (Captiflex Extra Small Oval - Flexible 11 $\mathrm{mm}$; Boston Scientific, Natick, MA, USA) with an electrosurgical attachment (Boston Scientific) was used. The snare was placed over the polyp in an effort to remove a small 1 to $2 \mathrm{~mm}$ margin of normal surrounding tissue. The lumen was partially deflated to reduce tension on the colonic mucosa. The snare was closed, and electrocautery using standard setting (forced coag. 2, 20W) was applied before cutting through the polyps.

\section{( $\bullet$ Video 1)}

2. Cold snare: The same type of snare and a similar procedure was used, except the electrosurgical attachment was excluded. The snare was closed to cut through the polyp, and the tissue was removed by suction. ( $\triangle$ Video 2 )

3. Cold biopsy forceps: All polypoid tissue was removed with standard forceps (Radial Jaw 4 Large Capacity with $2.8 \mathrm{~mm}$ needle; Boston Scientific) by repeatedly pinching the tissue until no polyp was visible.

If additional polyp removal with other devices other than that assigned was necessary, then the resection was considered a failure.

Following the retrieval of the polyp, the polypectomy base (referred to as the base tissue) was lifted by submucosal injection of 1 to $5 \mathrm{~mL}$ of normal saline using a standard endoscopic needle (clear Interject Single-Use Sclerotherapy Needle, 23 gaugex $240 \mathrm{~cm}$; Boston Scientific). This was followed by excision with a clear margin of 1 to $2 \mathrm{~mm}$ using the cold snare device. In the event that no tissue could be removed (e.g., if the original resection achieved a wide resection), at least four cold biopsies using forceps on the remaining margins were obtained. The primary polyp specimen and the specimen from the base of the polyp were placed in separate jars. A maximum of five diminutive polyps were removed per patient as part of the research. There was no limit to the number of polyps removed overall as part of the screening or surveillance colonoscopy.

Data were collected using case report forms and then transferred to Research Electronic Data Capture (REDCap), a secure, webbased application designed to support data capture for research studies [7]. A single-study pathologist (M.K.) reviewed all base tissue samples. Gold standards based on the World Health Organization criteria were used for the final histopathological diagnoses of both the retrieved polyps and biopsies of the polyp bases [8]. Adequacy of the resection of diminutive polyps was defined as no visible adenoma or hyperplastic tissue seen in the base tissue on histology.

\section{Video 2}

Removal of two diminutive polyps with cold snare polypectomy technique.

online content including video sequences viewable at:

www.thieme-connect.de 
Table 1 Patient demographic and procedure information.

\begin{tabular}{|l|l|}
\hline Variable & $\begin{array}{l}\text { Overall } \\
\text { (N=60 patients) }\end{array}$ \\
\hline Age at exam & $60.4(33.8,52.2,69.9,82.1)$ \\
\hline Sex (female) & $26(43 \%)$ \\
\hline Ethnicity (Hispanic/Latino) & $3(5 \%)$ \\
\hline Patient race & $54(90 \%)$ \\
\hline White & $5(8 \%)$ \\
\hline Black or African American & $1(2 \%)$ \\
\hline Other & $4(2,3,7,25)$ \\
\hline Time to cecum from insertion time (min) & $18(7,13,27,76)$ \\
\hline Time from cecum to scope out (min) & $25(10,19,34,90)$ \\
\hline $\begin{array}{l}\text { Time from insertion of scope to scope out } \\
\text { (min) }\end{array}$ & $13(22 \%)$ \\
\hline Exam type/extent & $47(78 \%)$ \\
\hline EGD and colonoscopy & $6(10 \%)$ \\
\hline Colonoscopy only & $54(90 \%)$ \\
\hline Total bowel prep score & \\
\hline $6-7$ &
\end{tabular}

Abbreviation: EGD, upper endoscopy.

Categorical variables are summarized as $\mathrm{n}(\%)$; continuous variables are mean (minimum, $25^{\text {th }}$ percentile; maximum, $75^{\text {th }}$ percentile).

1 Based on the Boston Bowel Preparation Scale Score.

Patients were followed in a routine manner by their referring physicians. Major adverse events (perforation, bleeding requiring transfusion, admission, or emergency department visit) were captured by review of the clinical record approximately 30 days after colonoscopy and by telephone calls to the patients at that time.

\section{Results}

Between 26 September 2013 and 31 October 2013, 60 patients were enrolled ( Table 1 ). Of this group, 34 patients (57\%) were male, the mean age was 60 (range 33 - 82), and 54 patients (90\%) were White. A total of 62 polyps were randomized from 37 patients and their characteristics are shown in $\bullet$ Table 2 . An inadvertent recall error led to the use of a method different from that assigned for 3 polyps, which resulted in 18 hot snare procedures, 23 cold snare procedures, and 21 cold biopsy forceps procedures. Since this was a small pilot study, analyses were based on grouping according to these modified assignments. The performing endoscopists needed to use alternative methods from those assigned for 3 of the 62 polyps: cold biopsy forceps procedure was used in addition to hot snare for 1 polyp; hot snare was not considered appropriate for the removal of a polyp and cold biopsy forceps method was used instead; and cold biopsy forceps approach was not considered appropriate for removal of a polyp and the cold snare was used instead. The mean polyp size was $3.6 \mathrm{~mm}$ (range $2-5 \mathrm{~mm}$ ). Of the 62 polyps, 37 (60\%) were adenomatous (32 tubular adenoma, 4 sessile serrated adenoma/ polyp, and 1 tubulovillous adenoma); 20 (32\%) were hyperplastic; and 5 (8\%) were classified as other non-neoplasia/nonhyperplasia. These latter 5 polyps were excluded post-randomization because the aim of the study was to assess complete resection of neoplastic and hyperplastic lesions. This left 57 polyps: 18 removed by hot snare, 21 removed by cold snare, and 18 removed by cold biopsy forceps. Tissue retrieval rates were $100 \%$ for each method.
Table 2 Information on the polyps ${ }^{1}$ from patients ${ }^{2}$.

\begin{tabular}{|c|c|}
\hline Variable & $\begin{array}{l}\text { Overall } \\
\left(\mathrm{N}=62 \text { polyps }{ }^{1}\right)\end{array}$ \\
\hline \multicolumn{2}{|l|}{ Polyp location } \\
\hline Cecum & $8(13 \%)$ \\
\hline Ascending & $20(32 \%)$ \\
\hline Hepatic flexure & $1(2 \%)$ \\
\hline Transverse & $7(11 \%)$ \\
\hline Descending & $5(8 \%)$ \\
\hline Sigmoid & $16(26 \%)$ \\
\hline Rectum & $5(8 \%)$ \\
\hline \multicolumn{2}{|l|}{ Polyp shape } \\
\hline Sessile & $55(89 \%)$ \\
\hline Flat-slightly raised & $4(6 \%)$ \\
\hline Flat-completely flat & $3(5 \%)$ \\
\hline \multicolumn{2}{|l|}{ Polyp size (mm) } \\
\hline 2 & $11(18 \%)$ \\
\hline 3 & $21(34 \%)$ \\
\hline 4 & $10(16 \%)$ \\
\hline 5 & $20(32 \%)$ \\
\hline $\begin{array}{l}\text { Number of snare passes/bites performed to } \\
\text { remove polyp }\end{array}$ & $1(1,1,2,6)$ \\
\hline Time of polypectomy removal (s) & $53(22,40,83,240)$ \\
\hline \multicolumn{2}{|l|}{ Histopathology of polyp } \\
\hline Hyperplastic & $20(32 \%)$ \\
\hline Other non-neoplasia & $5(8 \%)$ \\
\hline Adenoma & $32(52 \%)$ \\
\hline Sessile serrated & $4(6 \%)$ \\
\hline Tubulovillous & $1(2 \%)$ \\
\hline Base snared successfully with EMR technique & $17(27 \%)$ \\
\hline Time of base lift and removal (s) & $90(50,75,118,255)$ \\
\hline \multicolumn{2}{|l|}{ Number of passes/bites for base lift } \\
\hline 1 & $17(27 \%)$ \\
\hline 4 & $45(73 \%)$ \\
\hline \multicolumn{2}{|l|}{ Histopathology of base tissue } \\
\hline Other non-neoplasia & $57(92 \%)$ \\
\hline Adenoma & $3(5 \%)$ \\
\hline Sessile serrated & $2(4 \%)$ \\
\hline
\end{tabular}

Abbreviation: EMR, endoscopic mucosal resection.

Categorical variables are summarized as $\mathrm{n}(\%)$; continuous variables are mean

(minimum, $25^{\text {th }}$ percentile; maximum, $75^{\text {th }}$ percentile).

${ }^{1} \mathrm{~N}=62$.

${ }^{2} \mathrm{~N}=37$.

The base tissue of 5 of the 57 polyps was positive: 2 sessile serrated adenomas and 3 adenomas. Descriptive information on these 5 lesions is provided in Table 3 . This gave an overall incomplete polyp resection rate of $9 \%(95 \% \mathrm{CI} 3-19 \%)$. The incomplete resection rate was 5 of 37 (14\%) for adenomas and 0 of 20 (0\%) for hyperplastic polyps. Overall, the assigned resection method failed for 7 patients, for a failure rate of $12 \%$ (95\%CI $5-24 \%$ ). This included the need to use alternative resection methods. By study arm, the incomplete resection rates were 1 of 18 (6\%) for hot snare, 2 of 21 (10\%) for cold snare, and 2 of 18 (11\%) for cold biopsy forceps. The failure rates were 2 of 18 (11\%) for hot snare, 2 of 21 (10\%) for cold snare, and 3 of 18 (17\%) for cold biopsy forceps. The majority of polyp bases were removed with cold biopsy forceps $(73 \%, N=45)$ since most of the endoscopists did not feel that the saline-lift cold snare method was feasible or appropriate. No intraprocedural bleeding events necessitating therapeutic intervention occurred and no adverse events related to polypectomy were reported at the 30-day patient telephone follow-up. 
Table 3 Information on the five polyps with residual neoplasia at the polyp base.

\begin{tabular}{|c|c|c|c|c|c|}
\hline \multirow[t]{2}{*}{ Variable } & \multicolumn{5}{|l|}{ Polyp } \\
\hline & 1 & 2 & 3 & 4 & 5 \\
\hline Group & Cold snare & Cold snare & Cold forceps & Cold forceps & Cold forceps \\
\hline Polyp location & Ascending & Ascending & Ascending & Transverse & Cecum \\
\hline Polyp shape & Sessile & Sessile & Sessile & Sessile & Sessile \\
\hline Polyp size (mm) & 5 & 4 & 3 & 5 & 3 \\
\hline Number of snare passes/bites to remove polyp & 1 & 1 & 2 & 5 & 2 \\
\hline Time of polypectomy removal (s) & 41 & 122 & 59 & 115 & 40 \\
\hline Base snared successfully with EMR technique & Yes & No & No & Yes & Yes \\
\hline Time of base lift and removal (s) & 125 & 148 & 79 & 240 & 88 \\
\hline Number of passes/bites for base lift & 1 & 4 & 4 & 1 & 1 \\
\hline Histopathology of polyp & Adenoma & Adenoma & Adenoma & Sessile serrated & Sessile serrated \\
\hline Histopathology of base tissue & Adenoma & Adenoma & Adenoma & Sessile serrated & Sessile serrated \\
\hline
\end{tabular}

Abbreviation: EMR, endoscopic mucosal resection.

\section{Discussion}

Failure of colonoscopy to prevent CRCs can be caused by several factors, including incomplete polypectomy. This pilot study confirmed that approximately 1 in 10 diminutive polyps found on colonoscopy are incompletely resected by standard polypectomy methods. This study also established the feasibility of conducting a larger trial of similar design to compare the three methods of polyp resection with randomization at the polyp level. It also established the preferred method of assessing completeness of resection (e.g., cold biopsy forceps in a four-quadrant fashion instead of saline-lift cold snare of the polyp base). Because of the small, intentionally created sample size, direct comparisons between the incomplete resection rates of the techniques or time taken to remove the polyps or polyp bases were not performed. To date, our pilot study is one of the few, if any, comparing complete diminutive polypectomy resection rates among three commonly used techniques (e.g., hot snare, cold snare, and cold biopsy forceps polypectomy). Several prospective studies have evaluated the completeness of polypectomy for diminutive lesions and compared a combination of techniques, but none compared all three commonly used techniques in the same study. Efthymiou et al. demonstrated a surprisingly high estimated rate of incomplete resection of $61 \%$ (33 of 54) among diminutive polyps that were removed with cold biopsy forceps; the majority of these incompletely resected polyps were hyperplastic [4]. The polyp base was resected using endoscopic mucosal resection (EMR) with a 1- to 2-mm margin. In our pilot study, safety and feasibility issues concerning performing EMR for such a small polyp resulted in the majority of endoscopists preferring to sample the polyp base with cold biopsy forceps. Electrocautery is the main risk factor for adverse events related to polypectomy, with blended coagulation current and pure coagulation current, respectively, being associated with immediate and delayed postpolypectomy bleeding $[9,10]$. Furthermore, performing a salinelift of the polyp base was technically challenging and time-consuming, making it unsuitable for the daily clinical practice setting. In the study by Efthymiou et al., several of the EMR specimens (e.g., base of the polypectomy site) were nonretrievable, highlighting the technical difficulty associated with injecting the polyp base and then having to resect such a small sample of colonic tissue [4]. However, EMR does have the main advantage of being able to assess whether the residual polyp is at the deep or lateral margin of the polyp base; therefore, this was one major limitation to our current pilot study. In another prospective study by Pohl and colleagues, 11 gastroenterologists had an estimated incomplete polyp resection rate of $10 \%$ for polyps ranging from 5 to $20 \mathrm{~mm}$ [5]. All polyps were removed with hot snare cautery, whereas the cold biopsy forceps were used to obtain tissue from the polyp resection margin. However, this study only evaluated the completeness of resection of polyps $5 \mathrm{~mm}$ and larger. Most recently, Lee and colleagues found that cold snare polypectomy was superior for removal of these smaller polyps ( 93 vs. $76 \%, P=$ 0.009) when compared to cold forceps polypectomy in a singlecenter, randomized controlled trial [6]. However, a double-biopsy technique with cold biopsy forceps was used to remove polyps, whereas in our study the endoscopists had the liberty to take as many cuts from the polyp until complete eradication was achieved and confirmed endoscopically.

Of the five polyps that had residual neoplasia at the base, two were sessile serrated adenomas removed with the cold biopsy forceps technique. Sessile serrated polyps are not only becoming increasingly recognized by endoscopists and pathologists, but can be more difficult to resect completely and are a well-established etiology for many of these "missed" or "interval" cancers. Although no statistical comparisons could be made among the three polypectomy techniques due to small sample size, by expanding to a larger, multicenter study it is plausible to speculate that cold forceps may result in higher rates of incompletely resected sessile serrated lesions when compared to cold or hot snare methods.

\section{Conclusions \\ $\nabla$}

The conclusions that can be drawn from this pilot study are limited by its small sample size; however, the primary intent was that the information gained would facilitate the refinement of the design of a larger, multicenter trial. In summary, we established the feasibility of recruiting patients to a study that randomizes polyps to one of three common methods of polypectomy for diminutive polyps. A larger, multicenter randomized study is necessary to be able to determine which method(s) of polypectomy are associated with higher complete resection rates. Ultimately, guidelines on the management of diminutive polyps are needed. 
Competing interests: V. G., R. J. B., J. E. C., M. K., N. N. D., none. M.B.W., research funding from Boston Scientific Corporation, Olympus, Ninepoint Medical; consultant, Endochoice and Fujinon

\section{Acknowledgments}

The authors are grateful for assistance with the preparation of the manuscript from John R. Cangemi, Michael F. Picco, and Massimo Raimondo, Division of Gastroenterology and Hepatology, Mayo Clinic, Jacksonville, FL, USA; and Kelly Viola, Academic and Research Support, Mayo Clinic, Jacksonville, FL, USA.

\section{References}

1 Baxter NN, Goldwasser MA, Paszat LF et al. Association of colonoscopy and death from colorectal cancer. Ann Intern Med 2009; 150: 1-8

2 Baxter NN, Warren JL, Barrett MJ et al. Association between colonoscopy and colorectal cancer mortality in a US cohort according to site of cancer and colonoscopist specialty. J Clin Oncol 2012; 30: 2664-2669
3 Farrar WD, Sawhney MS, Nelson DB et al. Colorectal cancers found after a complete colonoscopy. Clin gastroenterol hepatol 2006; 4: 12591264

4 Efthymiou M, Taylor AC, Desmond PV et al. Biopsy forceps is inadequate for the resection of diminutive polyps. Endoscopy 2011; 43: 312-316

5 Pohl $\mathrm{H}$, Srivastava A, Bensen SP et al. Incomplete polyp resection during colonoscopy-results of the complete adenoma resection (CARE) study. Gastroenterology 2013; 144: 74-80 e71

6 Lee CK, Shim JJ, Jang JY. Cold snare polypectomy vs. cold forceps polypectomy using double-biopsy technique for removal of diminutive colorectal polyps: a prospective randomized study. Am J Gastroenterol 2013; 108: $1593-1600$

7 Harris PA, Taylor $R$, Thielke $R$ et al. Research electronic data capture (REDCap) - a metadata-driven methodology and workflow process for providing translational research informatics support. J Biomed Inform 2009; 42: 377-381

8 Bosman FT, Carneiro F (eds.) WHO classification of tumours of the digestive system. Berlin: Springer-Verlag; 2010

9 Hewett DG, Rex DK. Colonoscopy and diminutive polyps: hot or cold biopsy or snare? Do I send to pathology? Clin gastroenterol hepatol 2011; 9: 102 - 105

10 Fatima H, Rex DK. Minimizing endoscopic complications: colonoscopic polypectomy. Gastrointest Endosc Clin N Am 2007; 17: 145 -156, viii 